International Journal of Engineering \& Technology, 7 (4) (2018) 2309-2313
International Journal of Engineering \& Technology
SPC
Website: www.sciencepubco.com/index.php/IJET
doi: $10.14419 /$ ijet.v7it.16694
Research paper

\title{
A sporadic decomposition of Hankel structured matrix in logarithmic and wavelet domain for impulse noise removal
}

\author{
Baby Victoria.L ${ }^{1 *}$, Sathappan. $S^{3}$ \\ ${ }^{1}$ Research Scholar, Erode Arts and Science College, Erode, Tamilnadu \\ ${ }^{2}$ Associate Professor, Erode Arts and Science College, Erode, Tamilnadu \\ *Corresponding author E-mail:victoriaphd123@gmail.com
}

\begin{abstract}
Noise removal from the color images is the most significant and challenging task in image processing. Among different conventional filter methods, a robust Annihilating filter-based Low-rank Hankel matrix (r-ALOHA) approach was proposed as an impulse noise removal algorithm that uses the sparse and low-rank decomposition of a Hankel structured matrix to decompose the sparse impulse noise components from an original image. However, in this algorithm, the patch image was considered as it was sparse in the Fourier domain only. It requires an analysis of noise removal performance by considering the other transform domains. Hence in this article, the rALOHA can be extended into other transform domains such as log and exponential. In the log and exponential domain, the logarithmic and exponential functions are used for modeling the multiplicative noise model. But, this model is used only for positive outcomes. Therefore, wavelet transform domain is applied to the noise model that localizes an image pixel in both frequency and time domain simultaneously. Moreover, it separates the most vital information in a given image. Thus, it is feasible for obtaining a better approximation of the considered function using few coefficients. Finally, the experimental results show the performance effectiveness of the proposed algorithm.
\end{abstract}

Keywords:Noise Removal; Fourier Transform; Log-Exponential Transform; R-ALOHA; Wavelet Transform.

\section{Introduction}

Generally, noise is defined as any unwanted pixels in the original images that may be classified into two types such as blur noise and impulse noise. Mostly, images are corrupted by various types of noises during its acquisition or transmission [1]. The fat-tailed distribution or impulse noise or spike noise is the most wellknown among different noises. Impulse noise can occur as a malfunction result of detector pixels in a digital camera or memory elements in imaging hardware. Impulse noise can be further classified into salt-and-pepper noise and random valued noise. A saltand-pepper noise matches an extreme dynamic range of a pixel value [2]. In this case, noisy pixels can be quite easily detected by an Adaptive Median Filter (AMF). An image containing salt-andpepper noise can have dark pixels i.e., pepper in bright regions and bright pixels i.e., salt in dark regions. Random-Valued Impulse Noise (RVIN) occurs within the dynamic range of an image pixel and it cannot be easily detected by AMF.

In recent, removal of impulse noise is an active research area using image processing techniques. The main objective of noise removal technique is suppressing the noise. The filter can be applied successfully for reducing heavy noise with less computational complexity [3]. The noise is removed gradually to preserve the information. When noise is non-additive, linear filtering techniques are not effective in removing the impulse noise. This has led to the utilization of non-linear filter techniques [4]. Over the past decades, different techniques were applied for the noise removal process. A sparse representation is a signal decomposition on a very small set of components which are adapted to the observational data. The sparse-decomposition based denoising is much better at the trade-off between the preservation of information and the suppression of noise. However, the sparse decomposition is adapted to a noisy image thus separating information from noise still has less efficiency.

Among different techniques, sparse and low-rank decomposition of Hankel structured matrix was proposed for impulse noise removal [5]. This approach was proposed based on the Annihilating filter-based Low-rank Hankel Matrix (ALOHA). Thus, it was known as robust ALOHA (r-ALOHA) according to the observation that an image corrupted with the impulse noise can be modeled as sparse components whereas underlying image can be modeled using a low-rank Hankel structured matrix. The sparse and low-rank matrix decomposition problem was solved by alternating direction technique of multiplier approach including initial factorized matrices coming from a low-rank matrix-fitting algorithm. This algorithm was applied in a patch-by-patch fashion for adapting the local image statistics that have different spectral distributions. However, the spectrum of a noiseless image was assumed as sparse in the Fourier domain only whereas the other transform domains were not considered.

Hence in this article, r-ALOHA is extended in some other transfer domains such as log and exponential domain. Image restoration in log domain may simplify the multiplicative noise model using logarithmic and exponential functions. But, log exponential transform is only used for positive outcomes. Therefore, a wavelet domain is further introduced that localizes an image signal in both time and frequency with less computation complexity whereas 
Fourier transform localizes only in frequency. Thus, the proposed noise removal in other transform domain can improve the performance of noise removal.

The rest of the article is structured as follows: Section II provides the previous researches related to impulse noise removal techniques. Section III explains the proposed noise removal technique in brief. Section IV compares the performance of the proposed technique with the existing technique and Section V concludes the research work.

\section{Literature survey}

A weighted couple sparse representation model [6] was proposed to remove the impulse noise. In this method, the coding coefficients were provided by exploiting the complicated relationships between the reconstructed and the noisy images with more appropriate for recovering the noise-free images. Here, the image pixels were categorized into noise-free, slightly corrupted and heavily corrupted. After that, different data fidelity regularizations were applied to different pixels in order to further improve the denoising performance. By solving a weighted rank-one minimization problem, the dictionary was trained directly on the noisy raw data. However, it requires a combination of non-local selfsimilarity priors to preserve more noise-free images and texture information.

A novel denoising scheme [7] was proposed to restore the noisy pixels by using non-uniform sampling and supervised piecewise autoregressive modeling based super-resolution. In this scheme, the noisy pixels were equivalently estimated in groups by resolving a well-designed optimization problem that assumes the image structure as an important constraint. The other objective was a piecewise autoregressive model to utilize all noise-free pixels for supervising the training model and solving the optimization problem. On the other hand, the computational complexity was high. A low-rank prior in small oriented noise-free image patches [8] was proposed to remove the impulse noise. In this method, a lowrank matrix approximation was used to preserve the texture data in the optimally oriented path based on the consideration of an oriented patch as a matrix. Based on this prior, a single-patch method was introduced within a generalized joint low-rank and sparse matrix recovery method to simultaneously detect and eliminate non-pointwise random-valued impulse noise. Also, a weighting matrix was added to encode an initial estimation of the spatial noise distribution. Moreover, optimal noise-free image patches were estimated by using an accelerated proximal gradient method. However, high-level information in the images was not considered. A novel Sparsity-Ranking Edge-Preservation Filter (SREPF) [9] was proposed to remove high-density impulse noise in images. The initial process of SREPF according to the sparse matrix representation was used to predict the noisy candidates and decide the processing order of them via a ranking of noise-pixels sparsity in the working window. Then, a modified double Laplacian convolution was applied to confirm the truly noisy pixels and yield a directional mean for recovering them. Conversely, computational complexity must be maintained at a low level for the preservation of edges and removal of impulse noise.

A two-stage quaternion switching filter [10] was proposed to remove the impulse noise in the color images. In this method, an effective color distance estimation method was proposed by using quaternion representation. Here, the directional samples along with four directions were utilized by this filter based on the new color distance measure to classify the image pixels into noise-free and noisy pixels. The principle of peer group was modified and extended to the directional samples to further detect the noisy pixels whether they were corrupted by impulse noise or not. At last, a weighted vector median filter was used only on the noisy pixels to remove such noises from the images. But, the computational complexity was high.

A combination of adaptive Vector Median Filter (VMF) and weighted mean filter [11] was proposed to remove high-density impulse noise from color images. In this approach, the noisy and non-noisy pixels were classified according to the non-causal linear prediction error. The adaptive VMF was processed over the noisy pixel where the window size was adapted based on the availability of high-quality pixels. On the other hand, a non-noisy pixel was substituted with the weighted mean of high-quality pixels of the processing window. However, the computational complexity was high.

\section{Proposed methodology}

In this section, the proposed image modeling in the log, exponential transform namely e1-ALOHA and wavelet transforms namely e2-ALOHA is explained. Initially, the image is modeled by using sparse and low-rank decomposition model that constructs the Hankel structured matrix. Here, the proposed approach is used to obtain the noiseless image by considering the spectrum of that image is sparse in the log, exponential and wavelet domains i.e., the sparse components are derived by using log, exponential functions and wavelet functions which are described in below. In addition, the Hankel structured matrix is determined by noise-free image size and related annihilating filter size based on the optimization method such as pixel-by-pixel soft thresholding approach [5].

\subsection{Log exponential transform domain (e1-ALOHA)}

Based on the Hankel structured matrix $\mathcal{H}$, the image with impulse noise $\mathrm{M}$ can be modeled as:

$$
\mathcal{H}(\mathrm{M})=\mathrm{I}+\mathrm{S}(3.1)
$$

Equation (3.1), I refers to the noise-free image and $\mathrm{S}$ refers to the sparse matrix composed of impulse noise. By considering a sparse matrix, the image is modeled with minimum Total Variations (TV) which provides the following cost function:

$$
\|\mathrm{M}-\mathrm{I}\|_{1}+\lambda \mathrm{TV}(\mathrm{I})(3.2)
$$

Equation (3.2), $\|\cdot\|_{1}$ norm denotes the $l_{1}$ norm related to the sum of absolute values of each matrix element for removing outlier and TV(I) refers the 2D TV penalty in the image modeling. The proposed approach is nearly associated with an annihilating filter relationship from the sampling theory of signals with a Finite Rate of Innovations (FRI). When an image patch $\mathrm{x}[\mathrm{n}]$ in the discrete domain has sparse spectral components in the log domain, a corresponding annihilating filter exists in the image domain. The equation (3.1) can be modified by using a logarithmic transform on both sides as follows:

$$
\begin{array}{r}
\log \mathcal{H}(\mathrm{M})=\log \mathrm{I}+\log \mathrm{S}(3.3) \\
\mathrm{P}=\mathrm{J}+\mathrm{T}(3.4)
\end{array}
$$

Equation (3.4), P, J and $\mathrm{T}$ are the element-wise logarithms of $\mathcal{H}(\mathrm{M}), \mathrm{I}$ and $\mathrm{S}$, correspondingly. The function $\mathrm{S}=\mathrm{e}^{\mathrm{T}}$ is strictly monotonic and the elements of S satisfy the independent and identically distributed (i.i.d.) Gamma distribution. The Probability Density Function (PDF) of the elements in T is given by:

$$
\mathrm{f}_{\mathrm{T}}(\mathrm{T})=\prod_{\mathrm{x}=1}^{\mathrm{N}} \frac{\mathrm{L}^{\mathrm{L}}}{\Gamma(\mathrm{L})} \mathrm{e}^{\mathrm{L}\left(\mathrm{T}_{\mathrm{x}}-\mathrm{e}^{\mathrm{T}} \mathrm{x}\right)}(3.5)
$$

Equation (3.5), L refers to the positive integer used to define the noise level, $\Gamma(\cdot)$ refers to the Gamma function denoted by $\Gamma(\mathrm{L})=$ $(\mathrm{L}-1)$ ! and $\mathrm{T}_{\mathrm{X}}$ refers the elements of the vector $\mathrm{T}$ with the number of image patches $x=1,2, \ldots, n$. Thus, the log-likelihood function is represented as:

$$
\log f_{P \mid J}(P \mid J)=\log f_{T}(P-J)
$$




$$
=\mathrm{N} \log \frac{\mathrm{L}^{\mathrm{L}}}{\Gamma(\mathrm{L})}+\mathrm{L} \sum_{\mathrm{x}=1}^{\mathrm{n}} \mathrm{P}_{\mathrm{x}}-\mathrm{L} \sum_{\mathrm{x}=1}^{\mathrm{n}}\left(\mathrm{J}_{\mathrm{x}}+\mathrm{e}^{\mathrm{P}_{\mathrm{x}}-\mathrm{J}_{\mathrm{x}}}\right)(3.6)
$$

By maximizing the equation (3.6) with respect to J, the Maximum Likelihood (ML) estimation for J is achieved by following minimization problem:

$$
\hat{\mathrm{J}}=\arg \min _{\mathrm{J}} \sum_{\mathrm{x}=1}^{\mathrm{n}}\left(\mathrm{J}_{\mathrm{x}}+\mathrm{e}^{\mathrm{P}_{\mathrm{x}}-\mathrm{J}_{\mathrm{x}}}\right)(3.7)
$$

The optimal solution to the above problem is $\hat{\jmath}=\mathrm{P}$. Due to an over-fitting problem, this solution is not suitable for the denoising process. Hence, regularization technique is used for encouraging the sparsity and smoothness prior correspondingly. By combining regularization parameters, the new formulation is written as:

$$
\begin{array}{r}
\hat{\mathrm{J}}=\arg \min _{\mathrm{J}} \sum_{\mathrm{x}=1}^{\mathrm{n}} \sum_{\mathrm{y}=1}^{\mathrm{m}}\left(\mathrm{J}_{\mathrm{x}, \mathrm{y}}+\mathrm{e}^{\mathrm{P}_{\mathrm{x}, \mathrm{y}}-\mathrm{J}_{\mathrm{x}, \mathrm{y}}}\right)+\lambda_{1}\|\Omega \mathrm{J}\|_{1}+ \\
\lambda_{2} \mathrm{G}_{\beta}\{\mathrm{R}(\mathrm{J})\}(3.8)
\end{array}
$$

Equation (3.8), $\sum_{\mathrm{x}=1}^{\mathrm{n}} \sum_{\mathrm{y}=1}^{\mathrm{m}}\left(\mathrm{J}_{\mathrm{x}, \mathrm{y}}+\mathrm{e}^{\mathrm{P}_{\mathrm{x}, \mathrm{y}}-\mathrm{J}_{\mathrm{x}, \mathrm{y}}}\right)$ refers the data fidelity term which is estimated by using (3.7). The parameters $\lambda_{1}$ and $\lambda_{2}$ are the multipliers for balancing the data fidelity term and regularizations. The first regularization term $\|\Omega J\|_{1}$ reflects the sparse property of the image patches with respect to $\Omega$ which is known as dictionary learned from the image patches. The second regularization term $G_{\beta}\{R(J)\}$ is used for encouraging the smoothness of the entire image where the image patchJ is reshaped back to the complete image by applying the operator $\mathrm{R}(\cdot)$ and $\mathrm{G}_{\beta}\{\cdot\}$ refers the smoothness promotion function. For a given image $P \in \mathbb{R}^{\mathrm{d} \times \mathrm{d}}$, $\mathrm{G}_{\beta}\{\mathrm{P}\}$ is defined as:

$$
\mathrm{G}_{\beta}\{\mathrm{P}\}=\sum_{\mathrm{x}=1}^{\mathrm{d}} \sum_{\mathrm{y}=1}^{\mathrm{d}}\left(\sqrt{\left(\nabla_{\mathrm{h}} \mathrm{P}_{\mathrm{x}, \mathrm{y}}\right)^{2}+\left(\nabla_{\mathrm{v}} \mathrm{P}_{\mathrm{x}, \mathrm{y}}\right)^{2}}\right)^{\beta}
$$

Here, $\nabla_{h} P_{x, y}$ and $\nabla_{v} P_{x, y}$ are horizontal and vertical differences at pixelP $P_{x, y}$. The parameter $\beta$ controls the degree of smoothing. If the parameter $\lambda_{2}$ is zero, then the equation (3.8) can be rewritten as:

$$
\hat{\mathrm{J}}=\arg \min _{\mathrm{J}} \sum_{\mathrm{x}=1}^{\mathrm{n}} \sum_{\mathrm{y}=1}^{\mathrm{m}}\left(\mathrm{J}_{\mathrm{x}, \mathrm{y}}+\mathrm{e}^{\mathrm{P}_{\mathrm{x}, \mathrm{y}}-\mathrm{J}_{\mathrm{x}, \mathrm{y}}}\right)+\lambda\|\Omega \mathrm{J}\|_{1}(3.10)
$$

Furthermore, the restored log-image $\hat{J}$ is obtained by applying the operator $\mathrm{R}(\cdot)$ to the solution to (3.8), and the denoised image $\hat{I}$ is obtained by taking the exponential transform of $\hat{\jmath}$.

\subsection{Wavelet transform domain (e2-ALOHA)}

Initially, the noisy image $\mathcal{H}(\mathrm{M}(\mathrm{t}))$ is differentiated for obtaining the image $\mathcal{H}_{\mathrm{d}}(\mathrm{M}(\mathrm{t}))$ by using the central finite differences method with the fourth-order correction that minimizes the error as:

$$
\mathcal{H}_{\mathrm{d}}(\mathrm{M}(\mathrm{t}))=\frac{\mathrm{d} \mathcal{H}(\mathrm{M}(\mathrm{t}))}{\mathrm{dt}}(3.11)
$$

Then, the wavelet coefficients $\left(\mathrm{W}_{\mathrm{u}, \mathrm{v}}\right)$ are obtained at different dyadic scales $\mathrm{u}$ and displacements $\mathrm{v}$ by considering wavelet transform of the data $\mathcal{H}_{\mathrm{d}}(\mathrm{M}(\mathrm{t}))$. A dyadic scale is a scale whose numerical magnitude is equivalent to 2 rises to an integer exponent and labeled by the exponent. Therefore, the dyadic scale $u$ refers to a scale of size $2^{u}$. So, a low value of $u$ refers a finer resolution when high $u$ analyses the image at a larger resolution. This transform is the discrete wavelet transform and is given by:

$$
\mathrm{W}_{\mathrm{u}, \mathrm{v}}=\int_{-\infty}^{\infty} \mathcal{H}_{\mathrm{d}}(\mathrm{M}(\mathrm{t})) \psi_{\mathrm{u}, \mathrm{v}}(\mathrm{t}) \mathrm{dt}(3.12)
$$

Where $\psi_{\mathrm{u}, \mathrm{v}}(\mathrm{t})=2^{\mathrm{j} / 2} \psi\left(2^{\mathrm{j}} \mathrm{t}-\mathrm{v}\right)(3.13)$
Here, $u, v$ are integers. After that, the power $E_{u}$ in different dyadic scale $\mathrm{u}$ is estimated as:

$$
\mathrm{E}_{\mathrm{u}}(\mathcal{H}(\mathrm{M}))=\sum_{\mathrm{v}=-\infty}^{\infty}\left|\mathrm{W}_{\mathrm{u}, \mathrm{v}}\right|^{2}, \mathrm{u}=1,2, \ldots(3.1
$$

By plotting the deviation of $\mathrm{E}_{\mathrm{u}}$ with $\mathrm{u}$, it is possible to identify a scale $u_{m}$ at which the power due to noise falls off rapidly. This is significant since it provides an automated detection of the threshold. Identification of the scale $u_{m}$ at which the power due to noise demonstrates the first minimum that allows to rest all $W_{u, v}$ up to scale index $u_{m}$ to zero i.e., $\mathrm{W}_{\mathrm{u}, \mathrm{v}}=0$ for $\mathrm{u}=1,2, \ldots, \mathrm{u}_{\mathrm{m}}$. Moreover, the denoised data $\widehat{\mathcal{H}}_{\mathrm{d}}(\mathrm{M}(\mathrm{t}))$ is reconstructed by taking the inverse transform of the coefficients $\left(\mathrm{W}_{\mathrm{u}, \mathrm{v}}\right)$ as:

$$
\begin{array}{r}
\widehat{\mathcal{H}}_{\mathrm{d}}(\mathrm{M}(\mathrm{t}))=\mathrm{c}_{\psi} \sum_{\mathrm{u}=0}^{\infty} \sum_{\mathrm{v}=-\infty}^{\infty} \mathrm{W}_{\mathrm{u}, \mathrm{v}} \psi_{\mathrm{u}, \mathrm{v}}(\mathrm{t})(3.15) \\
\text { Where } \mathrm{c}_{\psi}=\frac{1}{\int_{-\infty}^{\infty} \frac{|\widehat{\Psi}(\omega)|^{2}}{\omega} \mathrm{d} \omega<\infty}(3.16)
\end{array}
$$

Here, $c_{\psi}$ refers to the normalization constant and $\widehat{\psi}(\omega)$ is the Fourier transform of the wavelet function $\psi(\mathrm{t})$. At last, the obtained $\widehat{\mathcal{H}}_{\mathrm{d}}(\mathrm{M}(\mathrm{t}))$ is integrated to yield the noise-free image $\hat{\mathrm{I}}(\mathrm{t})$ as:

$$
\hat{\mathrm{I}}(\mathrm{t})=\int \widehat{\mathcal{H}}_{\mathrm{d}}(\mathrm{M}(\mathrm{t})) \mathrm{dt}(3.17)
$$

Thus, the spectrum of a noiseless image patch is considered as a sparse in the log, exponential domain and wavelet transform domain efficiently.

\section{Result and discussion}

In this section, performance effectiveness of the proposed approaches named extended version-1 ALOHA (e1-ALOHA) and extended version-2 ALOHA (e2-ALOHA) are evaluated in MATLAB 2018a and compared with the existing approaches such as r-ALOHA and ALOHA. The comparison is carried out in terms of Peak Signal-to-Noise Ratio (PSNR), Structural Similarity Index Metric (SSIM) and reconstruction (computation) time for different images such as Lena, Barbara, balloon and cameraman at noise density level is $25 \%$.

\subsection{Peak signal-to-noise ratio (PSNR)}

PSNR is defined as the fraction of maximum possible signal power to the corrupting noise power which affects the fidelity of its representation. Generally, it is defined by using Mean Squared Error (MSE) and computed as:

$$
P S N R=10 \log _{10} \frac{255^{2}}{M S E}(4.1)
$$

Where, $M S E=\frac{1}{m n} \sum_{i=1}^{m-1} \sum_{j=1}^{n-1}\left(M_{i j}-I_{i j}\right)^{2}(4.2)$ 


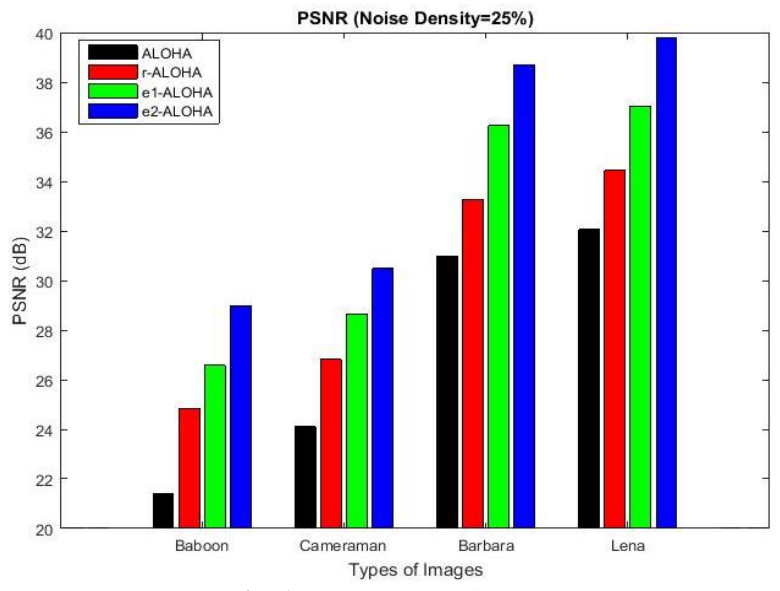

Fig. 1: Comparison of PSNR

Figure 1 shows the comparison of different approaches by considering the images such as baboon, cameraman, Barbara and Lena in terms of PSNR (dB) at noise density level is $25 \%$. The PSNR value of proposed e2-ALOHA is $7.4 \%$ higher than e1-ALOHA, $15.5 \%$ higher than r-ALOHA and $24.1 \%$ higher than ALOHA when Lena image is considered. From the analysis, it is observed that the e2-ALOHA has better PSNR than the other approaches.

\subsection{Reconstruction time}

It is defined as the time required for reconstructing the original image i.e., a time required to obtain the noise-free images from noisy images.

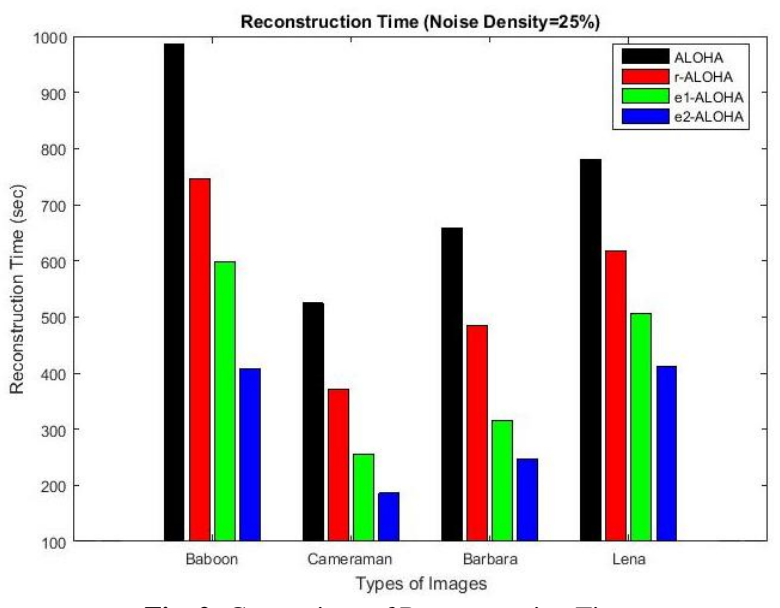

Fig. 2: Comparison of Reconstruction Time.

Figure 2 demonstrates the comparison of reconstruction time (seconds) for different approaches by considering various images like baboon, cameraman, Barbara and Lena at noise density level is $25 \%$. The reconstruction time value of proposed e2-ALOHA is $18.9 \%$ less than e1-ALOHA, $33.4 \%$ less than r-ALOHA and $47.4 \%$ less than ALOHA for considering Lena image. Through the analysis, it is that noticed that e2-ALOHA has reduced reconstruction (computation) time than the other approaches.

\subsection{Structural similarity index metric (SSIM)}

SSIM is defined as the similarity value between the original $M$ and denoised images $I$. It is computed as:

$$
\operatorname{SSIM}(x, y)=\frac{\left(2 \mu_{M} \mu_{I}+c_{1}\right)\left(2 \sigma_{M I}+c_{2}\right)}{\left(\mu_{M}^{2}+\mu_{I}^{2}+c_{1}\right)\left(\sigma_{M}^{2}+\sigma_{I}^{2}+c_{2}\right)}(4.3)
$$

Here, $\mu_{M}, \mu_{I}$ are averages and $\sigma_{M}^{2}, \sigma_{I}^{2}$ are variances of $\mathrm{M}$, I respectively, $\mathrm{c}_{1}, \mathrm{c}_{2}$ are constants and $\sigma_{\mathrm{MI}}$ is the covariance of $\mathrm{M}$ and I.

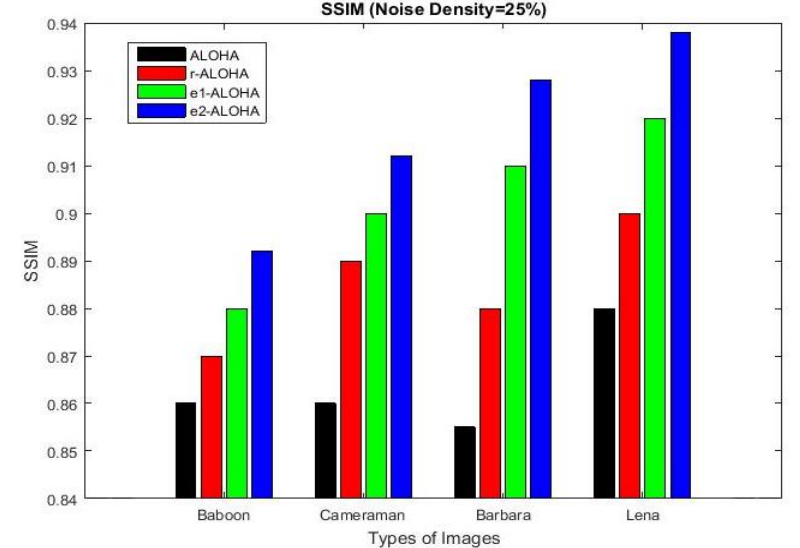

Fig. 3: Comparison of SSIM.

Figure 3 demonstrates the comparison of SSIM for different approaches by considering various images like baboon, cameraman, Barbara and Lena at noise density level is $25 \%$. The SSIM value of proposed e2-ALOHA is $1.96 \%$ higher than e1-ALOHA, $4.22 \%$ higher than r-ALOHA and 6.59\% higher than ALOHA when Lena image is considered. Through the analysis, it is that concluded that e2-ALOHA has improved SSIM than the other impulse noise removal approaches.

\section{Conclusion}

In this article, an enhanced robust ALOHA is proposed for impulse noise removal. According to the observation that smoothness or textures within an image patch correspond to sparse spectral components in the log, exponential transform domain and wavelet transform domain, the proposed ALOHA exploits the existence of annihilating filters and the associated rank-deficient Hankel matrices in an image domain for estimating any missing pixels. In this approach, impulse noise is modeled as sparse components and the image is modeled by using low-rank Hankel structured matrix. Also, the local image statistics that have individual spectral distributions is adapted by applying the proposed approach in a patch-by-patch manner. Finally, the experimental results prove that e2-ALOHA has better efficiency than the other approaches when noise density level is considered as $25 \%$. Similarly, the performance is increased when increasing the noise density level to $50 \%$ and $75 \%$ as well. In future, the other transform domains like hough/radon and gradient domain transform would be considered to analyze the performance of noise removal.

\section{References}

[1] Davis RR, \& Clavier O (2017), "Impulsive noise: A brief review", Hearing research, 349, 34-36. https://doi.org/10.1016/j.heares.2016.10.020.

[2] Koli M, \&Balaji S (2013), "Literature survey on impulse noise reduction”, Signal \& Image Processing, 4(5), 75.https://doi.org/10.5121/sipij.2013.4506.

[3] Suganthi A, \&Senthilmurugan M (2013), "Comparative study of various impulse noise reduction techniques", International Journal of Engineering Research and Applications, 3(5), 1302-1306.

[4] Pritamdas K, Singh KhM, \& Singh LL (2017), "A summary on various impulse noise removal techniques", International Journal of Science and Research, 6(3), 941-954.

[5] Jin KH, \& Ye JC (2018), "Sparse and low-rank decomposition of a hankel structured matrix for impulse noise removal", IEEE Transactions on Image Processing, 27(3), 14481461.https://doi.org/10.1109/TIP.2017.2771471.

[6] Chen CLP, Liu L, Chen L, Tang YY, \& Zhou Y (2015), "Weighted couple sparse representation with classified regularization for impulse noise removal", IEEE Transactions on Image Processing, 24(11), 4014-4026.https://doi.org/10.1109/TIP.2015.2456432.

[7] Wang X, Shi G, Zhang P, Wu J, Li F, Wang Y, \& Jiang H (2016), "High quality impulse noise removal via non-uniform sampling and 
autoregressive modelling based super-resolution", IET Image Processing, 10(4), 304-313.https://doi.org/10.1049/iet-ipr.2015.0216.

[8] Wang R, Pakleppa M, \&Trucco E (2015), "Low-rank prior in single patches for nonpointwise impulse noise removal", IEEE Transactions on Image Processing, 24(5), 1485 1496.https://doi.org/10.1109/TIP.2015.2400225.

[9] Chou HH, Lin HW, \& Chang JR (2014), "A sparsity-ranking edgepreservation filter for removal of high-density impulse noises", AEU-International Journal of Electronics and Communications, 68(11), 1129-1135.https://doi.org/10.1016/j.aeue.2014.06.001.

[10] Jin L, Zhu Z, Xu X, \& Li X (2016), "Two-stage quaternion switching vector filter for color impulse noise removal", Signal Processing, 128, 171-185.https://doi.org/10.1016/j.sigpro.2016.03.025.

[11] Roy A, Singha J, Manam L, \&Laskar RH (2017), "Combination of adaptive vector median filter and weighted mean filter for removal of high-density impulse noise from colour images", IET Image Processing, 11(6), 352-361.https://doi.org/10.1049/ietipr.2016.0320. 